\title{
Analysis of Reasons For Readmission of Diabetic Patients: 2015-2019
}

\author{
Junmei Wang \\ Yongchuan Hospital of Chongqing Medical University \\ Jiayao Xiong \\ Yongchuan Hospital of Chongqing Medical University \\ Chao Yang \\ Yongchuan Hospital of Chongqing Medical University \\ Xianshu Jiang \\ Yongchuan Hospital of Chongqing Medical University \\ Min Zhang \\ Yongchuan Hospital of Chongqing Medical University \\ Yanmei Chen \\ Yongchuan Hospital of Chongqing Medical University \\ Dezhi Chen \\ Yongchuan Hospital of Chongqing Medical University

\section{Zhiyong Li} \\ Yongchuan Hospital of Chongqing Medical University \\ Xiaolan Zhao ( $1210366394 @ q q . c o m$ ) \\ Yongchuan Hospital of Chongqing Medical University
}

\section{Research Article}

Keywords: diabetes mellitus, reasons for readmission, hospitalisation costs, preventive strategies

Posted Date: January 3rd, 2022

DOI: https://doi.org/10.21203/rs.3.rs-1199123/v1

License: (c) (i) This work is licensed under a Creative Commons Attribution 4.0 International License.

Read Full License 


\section{Abstract}

Background: Among patients with diabetes who had been hospitalized, $30 \%$ had twice or more hospitalisations rate, accounting for more than $50 \%$ of total hospitalizations and hospitalization expense. The purpose of our study was to to find available strategies to reduce the readmission rate of diabetics in rural areas.

Methods: This retrospective single-center study used the data from Yongchuan Hospital of Chongqing Medical University. The t-test and the chi-square test or Fisher's exact test were used to compare continuous and categorical variables, respectively. We used the Spearman correlation coefficient to examine the relationship between variables. Multiple linear regression was performed to analyze the influencing factors of hospitalisation time, and dummy variables were set for categorical independent variables.

Results: There were a total of 1721 readmissions during a five-year period; among them, 829 were females and 892 males. The readmission rate of diabetic patients in the endocrinology department was $32.40 \%$. The age, times of hospitalisation, and duration of all subjects were $64.67 \pm 13.82,2.69 \pm 1.41$ and $10.60 \pm 6.78$, respectively. Among all the diabetic patients, type 2 diabetes accounted for $98.55 \%(n=$ 1696). Most of the patients were readmitted due to poor glycemic control, infection, edema, dizziness, and weakness, accounting for approximately $56 \%$. During the 5 -year period, the majority of readmitted diabetic patients were hospitalized twice. Times of hospitalisation was weakly positively correlated with age $(\mathrm{Rho}=0.206, \mathrm{P} \leq 0.001)$, diabetic duration $(\mathrm{Rho}=0.248, \mathrm{P} \leq 0.001)$ and hospitalisation expenses (Rho $=0.008, P=0.035)$ by Spearman correlation analysis. Age, duration of diabetes, systolic blood pressure (SBP), diastolic blood pressure (DBP) and alanine aminotransferase (ALT) were the main factors affecting times of hospitalisation in diabetes patients (all $\mathrm{P}<0.05$ ). Compared with current smokers, nonsmokers and cessation smokers had high hospitalisations rate (all P for trend $<0.05$ ). When taking diabetic foot infection as a reference, edema was more accountable than diabetic foot infection for hospitalisation times, which was statistically significant $(P$ for trend $=0.048$ ).

Conclusion: Age, duration of diabetes and hospitalisation costs were positively correlated with times of hospitalisation. Age, duration of diabetes, blood pressure, ALT, smoking status and edema are the influencing factors of hospitalisation times. The most common causes of hospitalisation for diabetics

are poor glycemic control, infection, edema, dizziness, and weakness. Controlling these factors may be key to developing rational health strategies for rural diabetics.

\section{Introduction}

Diabetes, a chronic non-communicable disease, affects thousands of people all over the world. It is reported that there will be an estimated 693 million (9.9\%) adult diabetics globally by 2045 [1]. Due to urbanization, aging, increased prevalence of overweight and obesity and genetic susceptibility of Chinese 
people, the prevalence of diabetes in China is rapidly increasing. Presently, China has become the country with the most diabetes patients in the world.

Diabetes mellitus (DM) can be accompanied by acute and chronic complications, leading to organ dysfunction and failure, and even disability or death. DM is not only a serious health problem facing the world with high disability rate, morbidity and mortality, but also a huge economic burden on individuals, family and society. The total global healthcare expenditure due to diabetes was estimated to be USD 850 billion in 2017, and will increase by 7\% to USD 958 billion by 2045[1]. Analogously, the total annual medical cost of diabetes treatment in China was about 85-100 billion RMB (USD 12.93-15.21 billion) in 2013[2]. Nevertheless, the health systems in most of the low- or middle-income developing countries are not sufficiently equipped to handle the rapidly rising burden of diabetes [3].

Diabetic patients have a higher risk of hospitalisation than non-diabetic subjects, and a large proportion of these hospitalisations are due to diabetes-related complications [4-6]. Complications from diabetes are a serious threat to the healthcare system and one of the top ten reasons for readmission in public hospitals worldwide [7]. Among patients with diabetes who had been hospitalized, $30 \%$ had twice or more hospitalisations rate, accounting for more than $50 \%$ of total hospitalisations and hospitalisation expenses [8]. Therefore, this population-based study aims to analyze the causes of the rehospitalisation of diabetic patients, and proposed guidelines for treatment and follow-up to reduce the number of hospitalisations and the cost of health care.

\section{Methods}

\section{Definition}

We defined two or more hospitalisations as readmission.

\section{Procedures}

The data used in this study were obtained from the medical record department of Yongchuan Hospital of Chongqing Medical University, Chongqing China, a tertiary hospital. The principal diagnosis has been coded according to the Tenth International Classification of Diseases (ICD-10) of the World Health Organization at the time of discharge, the information of hospitalized diabetic patients in endocrinology department during the period from January 1, 2015 to December 31, 2019 is retrieved according to the main discharge diagnosis (E10, E11). As we utilized the principal discharge diagnosis to identify diabetic patients; therefore, those patients who were not primarily hospitalized for diabetes or transferred to the endocrinology department because of diabetes-related problems were excluded (Fig. 1). Namely, subjects were admitted and discharged from the endocrinology department. Likewise, patients who were admitted in 2014 and discharged in 2015 were excluded. We used Excel to screen out the information of patients who were hospitalized twice or more. The electronic medical record system was used to search the hospitalisation information according to the patient's hospitalisation number each time. The age, gender, diabetes duration, type of diabetes, smoking, body mass index (BMI), systolic blood pressure (SBP), 
diastolic blood pressure (DBP), glucose (Glu), hemoglobin A1c (HbA1c), triglyceride (TG), total cholesterol (TC), high density lipoprotein cholesterol (HDL-C), low density lipoprotein cholesterol (LDL-C), alanine aminotransferase(ALT), aspartate aminotransferase (AST), uric acid (UA), serum creatinine (Scr), times of hospitalisation, reasons for admission, hospitalisation costs, and treatment plans were extracted.

\section{Statistical analysis}

Descriptive statistics were used to describe clinical characteristics. Continuous variables were shown as Mean $\pm S D$, and t-test was used for comparison between groups. Categorical variables were expressed as frequency and percentage, and were compared using the chi-square test or the Fisher's exact test. Spearman correlation coefficient was applied to examine the relationship between variables. Multiple linear regression was performed to analyze the influencing factors of hospitalisation times, and dummy variables were set for categorical independent variables. A $P$ value $<0.05$ (two-tailed) was considered as statistically significant. All statistical analyses were performed with SPSS software version 26.0 (SPSS Inc., Chicago, IL).

\section{Results}

\section{Analysis of clinical characteristics}

During the study period, we totally screened 1721 readmissions with a principal diagnosis of DM (Fig. 1). Further, we calculated the rate of readmission for patients with DM in the endocrinology department was $32.40 \%$.

As shown in Table 1, a total of 829 females and 892 males were included in our study. The age, times of hospitalisation, and duration of all subjects were $64.67 \pm 13.82,2.69 \pm 1.41$ and $10.60 \pm 6.78$, respectively (Table 1). Among all the diabetic patients, type 2 diabetes mellitus accounted for $98.55 \%(n=$ 1696, Table 1). Interestingly, we found that both males and females did not smoke in the majority (69.61\%, Table 1). Compared with males, females had higher BMI, SBP, TC, HDL-C, and LDL-C $(P<0.05)$, and low levels of DBP, ALT, AST, UA, and Scr $(P<0.05$, Table 1). However, there was no significant difference in glucose, $\mathrm{HbA} 1 \mathrm{c}, \mathrm{TG}$, and hospitalisation costs in rehospitalisation diabetes patients among different gender (Table 1).

Our study found that 773 (44.92\%) patients were treated with insulin combined with non-insulin medicines in all readmissions (Table 1). Most of the patients were readmitted due to poor glycemic control, infection, edema, dizziness, and weakness, accounting for approximately 56\% (Table 1). During the 5-year period, most diabetic readmission patients were hospitalized twice (Fig. 2).

\section{The correlation of factors and times of hospitalisation}

The correlation between factors and times of hospitalisation were performed with Spearman correlation analysis. As shown in Table 2, times of hospitalisation were weakly positively 
correlated with age $(\mathrm{Rho}=0.206, \mathrm{P} \leq 0.001)$, diabetic duration $(\mathrm{Rho}=0.248, \mathrm{P} \leq 0.001)$ and hospitalisation expenses $(\mathrm{Rho}=0.008, \mathrm{P}=0.035)$.

\section{Association between other variables and times of hospitalisation}

Multiple linear regression was used to investigate the independent correlation between other variables and times of hospitalisation. As shown in Table 3, we found that age, duration of diabetes, SBP, DBP and ALT were the main factors affecting times of hospitalisation in diabetes patients (all $P<0.05$ ). The results showed that there was no statistical difference in the number of hospitalisations between type 1 and type 2 diabetic patients ( $P$ for trend $=0.859$, Table 4 ), male and female subjects ( $P$ for trend $=0.880$, Table 4). Surprisingly, compared with current smokers, non-smokers and cessation smokers had higher hospitalisations rate (all $\mathrm{P}$ for trend $<0.05$, Table 4 ).

With insulin treatment as the reference, non-insulin medicines (NIM) treatment, insulin+ NIM treatment and GS (glucose solution) treatment had no statistically significant effect on the number of hospitalisations (all P for trend $>0.05$, Table 4).

When taking diabetic foot infection as a reference, edema was more accountable than diabetic foot infection for hospitalisation times, which was statistically significant ( $P$ for trend $=0.048$, Table 4 ). However, there was no significant difference in the number of hospitalisations for poor glycemic control, diabetic ketoacidosis (DKA), hyperosmolar hyperglycemic syndrome (HHS), hypoglycemia, infection, microalbuminuria, blurred vision, dizziness, headache, chest distress, precordial discomfort, palpitation, dyspnea, chest pain, extremities pain, arthralgia, numbness, paresthesia, low back pain, consciousness disorders, abdominal distention, abdominal pain, vomiting, diarrhea, constipation, urinary incontinence, melena, pruritus, weakness, hepatic dysfunction, elevated blood glucose, and the classic symptoms of DM with diabetic foot infection (all P for trend $>0.05$, Table 4).

\section{Discussion}

The burden of hospitalized diabetics is huge, rapidly growing and expensive, and readmission is another large part of the burden [9]. In view of the significant impact on cost and quality, the hospital readmission rate is increasingly used as an indicator of health care quality and a potential source of regulatory penalties for hospitals [10]. Therefore, some countries use 30-days readmission rates to assess the quality of health care services. However, to the best of our knowledge, the readmission rate has not been taken as a measure of medical quality in China. Moreover, there is a lack of studies to investigate the diabetes readmission in China.

Our results revealed that the readmission rate of diabetic patients in the department of endocrinology at Yongchuan Hospital of Chongqing Medical University was 32.40\% in 5 years, which is consistent with Friedman's research that about one-third of diabetic patients had twice or more hospitalisations [11]. Overall, the reason why smokers and non-smokers are readmitted differently is that almost all female 
patients do not smoke. Furthermore, our study indicated that the most common causes of readmission for patients with DM were poor glycemic control, infection, edema, dizziness, and weakness.

Due to poor blood glucose control can lead to infection and other complications, need hospital treatment, so it is one of the main reasons for readmission. Such as edema, dizziness, weakness are symptoms of chronic complications of diabetes. These symptoms indicate long duration. Our results also show that duration related to the number of hospital, so there are the main reasons for rehospitalisation.

This study findings are consistent with those of Donnan et al [12] and Strack et al [13], that the readmission rate increases with age. Meanwhile, we also found that the duration of diabetes and hospitalisation costs were positively correlated with times of hospitalisation. Therefore, better glucose control reduction of complications and the times of hospitalisation are the key to reduce the burden of diabetes patients.

By multiple linear regression analysis, not only age and course of disease affected the number of hospitalisation, but it is also affected by blood pressure, ALT and smoking status. Moreover, the edema also affects times of hospitalisation. Although age is immutable, there may be other things they can reduce the readmission of diabetics, such as diabetes education, diabetes self-management, moderate exercise, smoking cessation, blood pressure control and so on.

A series of factors increase hospital readmission among patients with diabetes. Numerous studies that have investigated the risk factors for readmission of patients with diabetes demonstrate gender [14, 15], race $[8,9]$, insurance type[8, 15], $[9,13]$ urgent or emergent, comorbidity burden[8]. We did not collect some factors and our study period was different; therefore, we could not compare them with previous studies. However, our study helps to develop strategies for risk factors to reduce readmission.

As previous studies have shown that 27 percent of readmissions within 30 days are preventable [16], efforts are needed to reduce this frequency, especially among the elderly. Our findings show that multiple hospitalisations do not significantly change the health status of patients with diabetes, but may increase the number of readmissions. Similarly, adequate discharge planning, patient education, and follow-up actions are most effective in reducing readmission, which is consistent with previous studies [17-19]. To reduce readmission rates, we could create structured of individualized discharge plan for each patient with diabetes, as the American Diabetes Association does [20], but we have to be aware of the fact that they rarely follow this plan seriously. Hence, primary medical institutions play an important role in diabetes ambulatory management [21], and the family physician model may make a significant contribution to reducing the readmission rate of diabetic patients. For developing countries, these findings may help to plan future health strategies for diabetes management.

The study has several limitations that should be noted. First, this is a retrospective, observational, singlecenter study of outcomes data. Second, the readmission rate may be underestimated due to the fact that patients may be hospitalized in another medical institution where the hospitalisation records are not available in our hospital database, and patients die during our study period. Third, due to the limitation of 
China's medical insurance, people who are hospitalized for more than 30 days need to be readmitted. Therefore, it is possible to overestimate the number of readmissions.

\section{Conclusion}

We found that age, duration of diabetes and hospitalisation costs were positively correlated with times of hospitalisation. Age, duration of diabetes, blood pressure, ALT, smoking status and edema were the influencing factors of hospitalisation times. The most common causes of hospitalisation for diabetics, including poor glycemic control, infection, edema, dizziness, and weakness. The analysis of readmission of diabetic patients can help to plan the health strategy of diabetes management in the future, so as to reduce the readmission rate and the cost of health care.

\section{Abbreviations}

DM: Diabetes mellitus; ICD-10: the Tenth International Classification of Diseases; BMI: Body mass index; SBP: Systolic blood pressure; DBP: Diastolic blood pressure; Glu: Glucose; HbA1c: Hemoglobin A1c; TG: Triglyceride; TC: Total cholesterol; HDL-C: High density lipoprotein cholesterol; LDL-C: Low density lipoprotein cholesterol; ALT: Alanine aminotransferase; AST: Aspartate aminotransferase; UA: Uric acid; Scr: Serum creatinine; NIM: Non-insulin medicines; GS: Glucose solution; DKA: Diabetic ketoacidosis; HHS: Hyperosmolar hyperglycemic syndrome.

\section{Declarations}

\section{Acknowledgments}

We thank all participants of this program.

\section{Authors' contributions}

XLZ designed the study, reviewed and edited the article. ZYL supervised the work. JMW, MZ, YMC and DZC collected clinical data. JMW, JYX, CY and XSJ performed the statistical analysis. JMW wrote the manuscript. All authors have read and approved the fnal manuscript.

\section{Funding}

This research did not receive any specific grant from funding agencies in the public, commercial, or notfor-profit sectors.

\section{Availability of data and materials}

The datasets used and/or analysed during the current study are available from the corresponding author on reasonable request. 


\section{Ethics approval and consent to participate}

The study protocol was approved by the institutional review board of Yongchuan Hospital of Chongqing Medical University. All subjects gave written informed consent, and the study was conducted in accordance with the Declaration of Helsinki.

\section{Consent for publication}

Not applicable.

\section{Competing interests}

The authors declare that they have no competing interests.

\section{Author details}

Department of Endocrinology, Yongchuan Hospital of Chongqing Medical University, Yongchuan 402160, Chongqing, China.

\section{References}

1. Cho NH, Shaw JE, Karuranga S, et al. IDF Diabetes Atlas: Global estimates of diabetes prevalence for 2017 and projections for 2045. Diabetes Res Clin Pract. 2018;138:271-81.

2. Xu N, Liu KJ, Gu XF, et al. The research on total medical expenses of patients with diabetes. Chinese Health Economics. 2016;35:65-8.

3. Chen X, Yang W. Epidemic trend of diabetes in China: For the Xiaoren Pan Distinguished Research Award in AASD. J Diabetes Investig. 2014;5:478-81.

4. Mata-Cases M, Casajuana M, Franch-Nadal J, et al. Direct medical costs attributable to type 2 diabetes mellitus: a population-based study in Catalonia, Spain. Eur J Health Econ. 2016;17:1001-10.

5. Ostling S, Wyckoff J, Ciarkowski SL, et al. The relationship between diabetes mellitus and 30-day readmission rates. Clin Diabetes Endocrinol. 2017;3:3.

6. Tomlin AM, Tilyard MW, Dovey SM, et al. Hospital admissions in diabetic and non-diabetic patients: a case-control study. Diabetes Res Clin Pract. 2006;73:260-7.

7. Wong EL, Cheung AW, Leung MC, et al. Unplanned readmission rates, length of hospital stay, mortality, and medical costs of ten common medical conditions: a retrospective analysis of Hong Kong hospital data. BMC Health Serv Res. 2011;11:149.

8. Jiang HJ, Stryer D, Friedman B, et al. Multiple hospitalizations for patients with diabetes. Diabetes Care. 2003;26:1421-6. 
9. Rubin DJ. Hospital readmission of patients with diabetes. Curr Diab Rep. 2015;15:17.

10. Benbassat $\mathrm{J}$, Taragin M. Hospital readmissions as a measure of quality of health care: advantages and limitations. Arch Intern Med. 2000;160:1074-81.

11. Friedman B, Jiang HJ, Elixhauser A. Costly hospital readmissions and complex chronic illness. Inquiry. 2008;45:408-21.

12. Donnan PT, Leese GP, Morris AD. Hospitalizations for people with type 1 and type 2 diabetes compared with the nondiabetic population of Tayside, Scotland: a retrospective cohort study of resource use. Diabetes Care. 2000;23:1774-9.

13. Strack B, DeShazo JP, Gennings C, et al. Impact of $\mathrm{HbA} 1 \mathrm{c}$ measurement on hospital readmission rates: analysis of 70,000 clinical database patient records. Biomed Res Int. 2014;2014:781670.

14. Albrecht JS, Hirshon JM, Goldberg R, et al. Serious mental illness and acute hospital readmission in diabetic patients. Am J Med Qual. 2012;27:503-8.

15. Robbins JM, Webb DA. Diagnosing diabetes and preventing rehospitalizations: the urban diabetes study. Med Care. 2006;44:292-6.

16. van Walraven C, Bennett C, Jennings A, et al. Proportion of hospital readmissions deemed avoidable: a systematic review. Cmaj. 2011;183:E391-402.

17. Healy SJ, Black D, Harris C, et al. Inpatient diabetes education is associated with less frequent hospital readmission among patients with poor glycemic control. Diabetes Care. 2013;36:2960-7.

18. Misky GJ, Wald HL, Coleman EA. Post-hospitalization transitions: Examining the effects of timing of primary care provider follow-up. J Hosp Med. 2010;5:392-7.

19. Vernon D, Brown JE, Griffiths E, et al. Reducing readmission rates through a discharge follow-up service. Future Healthc J. 2019;6:114-7.

20. American Diabetes Association. 14. Diabetes Care in the Hospital: Standards of Medical Care in

Diabetes-2018. Diabetes Care. 2018;41:S144-51.

21. Reyes García R, Bernal Sobrino JL, Fernandez Pérez C, et al. Trends on Diabetes Mellitus's healthcare management in Spain 2007-2015. Diabetes Res Clin Pract. 2019;156:107824.

\section{Tables}

Table 1 Clinical characteristics of readmitted diabetic patients 


\begin{tabular}{|c|c|c|c|c|c|}
\hline & $\begin{array}{l}\text { All subjects } \\
(n=1721)\end{array}$ & $\begin{array}{l}\text { Female } \\
(n=829)\end{array}$ & $\begin{array}{l}\text { Male } \\
(n=892)\end{array}$ & $t / c 2$ & $p$ \\
\hline Age (years) & $\begin{array}{l}64.67 \pm \\
13.82\end{array}$ & $\begin{array}{l}65.99 \\
12.95\end{array}$ & $63.46 \pm 14.50$ & 2.329 & 0.020 \\
\hline Times & $2.69 \pm 1.41$ & $2.70 \pm 1.37$ & $2.69 \pm 1.44$ & 0.151 & 0.880 \\
\hline Type $(n, \%)$ & & & & 1.422 & 0.233 \\
\hline 1 & $25(1.45)$ & $15(1.81)$ & $10(1.12)$ & & \\
\hline 2 & $\begin{array}{l}1696 \\
(98.55)\end{array}$ & 814 (98.19) & $882(98.88)$ & & \\
\hline Duration (years) & $10.60 \pm 6.78$ & $11.43 \pm 7.12$ & $9.83 \pm 6.36$ & 2.984 & 0.003 \\
\hline Smoking $(n, \%)$ & & & & 687.259 & $<0.001$ \\
\hline Yes & 311 (18.07) & 0 & $311(34.87)$ & & \\
\hline No & $\begin{array}{l}1198 \\
(69.61)\end{array}$ & 827 (99.76) & 371 (41.59) & & \\
\hline Cessation & $212(12.32)$ & $2(0.24)$ & $210(23.54)$ & & \\
\hline $\mathrm{BMI}\left(\mathrm{Kg} / \mathrm{m}^{2}\right)$ & $24.34 \pm 3.69$ & $24.57 \pm 3.99$ & $24.12 \pm 3.37$ & 2.514 & 0.012 \\
\hline $\mathrm{SBP}(\mathrm{mmHg})$ & $\begin{array}{l}137.65 \pm \\
21.90\end{array}$ & $\begin{array}{l}138.80 \pm \\
22.10\end{array}$ & $\begin{array}{l}136.59 \pm \\
21.66\end{array}$ & 2.101 & 0.036 \\
\hline $\mathrm{DBP}(\mathrm{mmHg})$ & $\begin{array}{l}79.08 \pm \\
12.95\end{array}$ & $\begin{array}{l}77.78 \pm \\
12.36\end{array}$ & $80.30 \pm 13.37$ & -4.042 & $<0.001$ \\
\hline Glu (mmol/L) & $14.05 \pm 7.82$ & $13.91 \pm 7.87$ & $14.18 \pm 7.78$ & -0.722 & 0.470 \\
\hline $\mathrm{HbA1c}(\%)$ & $8.95 \pm 2.31$ & $9.01 \pm 2.34$ & $8.89 \pm 2.28$ & 1.096 & 0.273 \\
\hline TG (mmol/L) & $2.34 \pm 2.38$ & $2.33 \pm 2.07$ & $2.36 \pm 2.63$ & -0.259 & 0.796 \\
\hline $\mathrm{TC}(\mathrm{mmol} / \mathrm{L})$ & $4.75 \pm 1.62$ & $5.03 \pm 1.59$ & $4.49 \pm 1.61$ & 7.000 & $<0.001$ \\
\hline $\mathrm{HDL}-\mathrm{C}(\mathrm{mmol} / \mathrm{L})$ & $1.32 \pm 0.46$ & $1.41 \pm 0.45$ & $1.23 \pm 0.46$ & 8.394 & $<0.001$ \\
\hline LDL-C (mmol/L) & $2.54 \pm 1.13$ & $2.70 \pm 1.15$ & $2.39 \pm 1.09$ & 5.727 & $<0.001$ \\
\hline ALT (U/L) & $\begin{array}{l}28.02 \pm \\
55.08\end{array}$ & $\begin{array}{l}23.66 \pm \\
28.64\end{array}$ & $32.06 \pm 71.14$ & -3.254 & 0.001 \\
\hline AST (U/L) & $\begin{array}{l}27.65 \pm \\
52.32\end{array}$ & $\begin{array}{l}24.84 \pm \\
30.70\end{array}$ & $30.26 \pm 66.28$ & -2.204 & 0.028 \\
\hline $\mathrm{UA}(\mathrm{mmol} / \mathrm{L})$ & $\begin{array}{l}340.40 \pm \\
126.03\end{array}$ & $\begin{array}{l}323.43 \pm \\
121.81\end{array}$ & $\begin{array}{l}356.17 \pm \\
127.88\end{array}$ & -5.428 & $<0.001$ \\
\hline $\operatorname{Scr}(\mathrm{mmol} / \mathrm{L})$ & $\begin{array}{l}90.05 \pm \\
79.91\end{array}$ & $\begin{array}{l}80.21 \pm \\
60.85\end{array}$ & $99.20 \pm 93.34$ & -5.032 & $<0.001$ \\
\hline
\end{tabular}




\begin{tabular}{|c|c|c|c|c|c|}
\hline Cost (RMB) & $\begin{array}{l}15030.2 \pm \\
9095.21\end{array}$ & $\begin{array}{l}14614.4 \pm \\
8059.27\end{array}$ & $\begin{array}{l}15416.74 \pm \\
9950.86\end{array}$ & -1.844 & 0.065 \\
\hline Treatment $(n, \%)$ & & & & 口 & $0.531^{a}$ \\
\hline Insulin & $648(37.65)$ & 318 (38.36) & $330(37.00)$ & & \\
\hline NIM & $288(16.73)$ & $146(17.61)$ & $142(15.92)$ & & \\
\hline Insulin+ NIM & $773(44.92)$ & $362(43.67)$ & $411(46.08)$ & & \\
\hline GS & $8(0.46)$ & $2(0.24)$ & $6(0.67)$ & & \\
\hline GS+ Insulin & $1(0.06)$ & 0 & $1(0.11)$ & & \\
\hline GS+ NIM & $1(0.06)$ & 0 & $1(0.11)$ & & \\
\hline No treatment & $2(0.12)$ & $1(0.12)$ & $1(0.11)$ & & \\
\hline Reasons (n, \%) & & & & प & $<0.001^{\mathrm{a}}$ \\
\hline Poor glycemic control & $153(8.89)$ & 49 (5.91) & $104(11.66)$ & & \\
\hline DKA & $11(0.64)$ & $8(0.97)$ & $3(0.34)$ & & \\
\hline HHS & $3(0.17)$ & $2(0.24)$ & $1(0.11)$ & & \\
\hline \multicolumn{6}{|l|}{ Table 1 Cont. } \\
\hline & $\begin{array}{l}\text { All subjects } \\
(n=1721)\end{array}$ & $\begin{array}{l}\text { Female } \\
(n=829)\end{array}$ & $\begin{array}{l}\text { Male } \\
(n=892)\end{array}$ & $t / c 2$ & $p$ \\
\hline Hypoglycemia & $34(1.98)$ & $13(1.57)$ & $21(2.35)$ & & \\
\hline Infection & $278(16.15)$ & 145 (17.49) & $133(14.91)$ & & \\
\hline Microalbuminuria & $5(0.29)$ & $2(0.24)$ & $3(0.34)$ & & \\
\hline Edema & $119(6.91)$ & $49(5.91)$ & $70(7.85)$ & & \\
\hline Blurred vision & $45(2.61)$ & $29(3.50)$ & $16(1.79)$ & & \\
\hline Eye pain & $2(0.12)$ & $2(0.24)$ & 0 & & \\
\hline Dizziness & $268(15.57)$ & $158(19.06)$ & $110(12.33)$ & & \\
\hline Headache & $19(1.10)$ & $13(1.57)$ & $6(0.67)$ & & \\
\hline Chest distress & $12(0.70)$ & $5(0.60)$ & $7(0.78)$ & & \\
\hline Precordial discomfort & $7(0.41)$ & $7(0.84)$ & 0 & & \\
\hline Palpitation & $28(1.63)$ & $17(2.05)$ & $11(1.23)$ & & \\
\hline Dyspnea & $41(2.38)$ & $21(2.53)$ & $20(2.24)$ & & \\
\hline
\end{tabular}




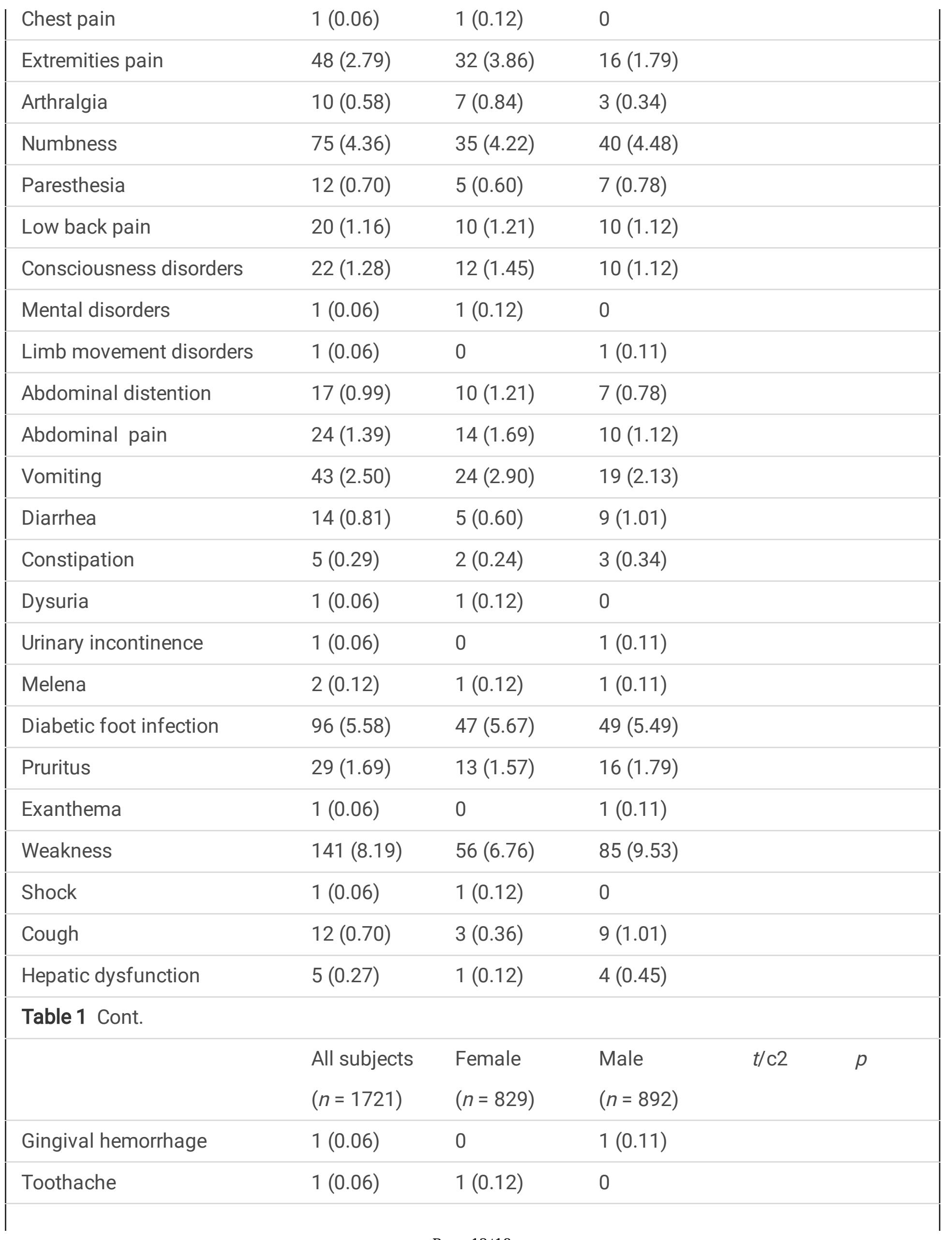




\begin{tabular}{llll} 
Appetite loss & $2(0.12)$ & $1(0.12)$ & $1(0.11)$ \\
\hline Elevated blood glucose & $8(0.46)$ & $3(0.36)$ & $5(0.56)$ \\
$\begin{array}{l}\text { The classic symptoms of } \\
\text { diabetes mellitus }\end{array}$ & $102(5.93)$ & $23(2.77)$ & $79(8.86)$
\end{tabular}

BMI, body mass index; SBP, systolic blood pressure; DBP, diastolic blood pressure; Glu, glucose; HbA1c, hemoglobin A1c; TG, triglyceride; TC, total cholesterol; HDL-C, high density lipoprotein cholesterol; LDL-C, low density lipoprotein cholesterol; ALT, alanine aminotransferase; AST, aspartate aminotransferase; UA, uric acid; Scr serum creatinine; NIM, non-insulin medicines; GS, glucose solution; DKA, diabetic ketoacidosis; HHS, hyperosmolar hyperglycemic syndrome; The classic symptoms of diabetes mellitus, polyuria, polydipsia, polyphagia, and weight loss. 1 RMB 0.1521 USD. Continuous variables were shown as mean \pm standard deviation, and the $t$-test was used for comparison between groups. Categorical variables were expressed as frequency and percentage, and were compared using the chi-square test or the Fisher's exact test. a, the Fisher's exact test.

Table 2 Spearman correlation analysis of factors for times of hospitalization

\begin{tabular}{cccccccc}
\hline Times & Age & Type & Duration & Smoking & Gender & BMI & SBP \\
\hline$r$ & $0.206^{* *}$ & -0.049 & $0.248^{* *}$ & 0.059 & -0.016 & 0.019 & 0.050 \\
$p$ & $<0.001$ & 0.215 & $<0.001$ & 0.139 & 0.685 & 0.623 & 0.207 \\
\hline
\end{tabular}

Table 2 Cont.

\begin{tabular}{cccccccc}
\hline Times & DBP & Glu & HbA1c & TG & TC & HDL-C & LDL-C \\
\hline$r$ & -0.009 & 0.003 & -0.008 & 0.004 & -0.007 & -0.049 & -0.019 \\
$p$ & 0.828 & 0.935 & 0.833 & 0.926 & 0.857 & 0.219 & 0.640 \\
\hline
\end{tabular}

Table 2 Cont.

\begin{tabular}{cccccccc}
\hline Times & ALT & AST & UA & Scr & Cost & Treatment & Reasons \\
\hline$r$ & 0.026 & -0.050 & -0.028 & 0.003 & 0.008 & -0.032 & -0.028 \\
$p$ & 0.520 & 0.210 & 0.482 & 0.939 & 0.035 & 0.426 & 0.483 \\
\hline
\end{tabular}

**P $\mathrm{P}<0.01$.

Table 3 Association between other continuous variables and times of hospitalization by multiple linear regression 


\begin{tabular}{|llllllll|}
\hline Variables & $\mathrm{B}$ & $\mathrm{SE}$ & $\mathrm{BC}$ & $t$ & $p$ & Lower $95 \% \mathrm{Cl}$ & Upper 95\%Cl \\
\hline Age & 0.018 & 0.004 & 0.176 & 4.314 & $<0.001$ & 0.010 & 0.026 \\
\hline Duration & 0.031 & 0.008 & 0.149 & 3.669 & $<0.001$ & 0.014 & 0.047 \\
\hline BMI & -0.007 & 0.016 & -0.018 & -0.451 & 0.652 & -0.038 & 0.024 \\
\hline SBP & 0.006 & 0.003 & 0.096 & 2.020 & 0.044 & $<0.001$ & 0.011 \\
\hline DBP & -0.014 & 0.005 & -0.130 & -2.803 & 0.005 & -0.024 & -0.004 \\
\hline Glu & -0.005 & 0.008 & -0.027 & -0.579 & 0.562 & -0.020 & 0.011 \\
\hline HbA1c & 0.018 & 0.028 & 0.028 & 0.621 & 0.535 & -0.038 & 0.073 \\
\hline TG & -0.009 & 0.033 & -0.014 & -0.284 & 0.777 & -0.074 & 0.055 \\
\hline TC & 0.037 & 0.077 & 0.042 & 0.480 & 0.631 & -0.114 & 0.188 \\
\hline HDL-C & -0.106 & 0.138 & -0.034 & -0.765 & 0.444 & -0.376 & 0.165 \\
\hline LDL-C & 0.026 & 0.097 & 0.021 & 0.273 & 0.785 & -0.164 & 0.217 \\
\hline ALT & 0.007 & 0.003 & 0.169 & 1.995 & 0.046 & $<0.001$ & 0.014 \\
\hline AST & -0.004 & 0.004 & -0.085 & -1.010 & 0.313 & -0.011 & 0.003 \\
\hline UA & $<0.001$ & $<0.001$ & -0.020 & -0.476 & 0.634 & -0.001 & 0.001 \\
\hline Scr & 0.001 & 0.001 & 0.041 & 0.963 & 0.336 & -0.001 & 0.002 \\
\hline Cost & $<0.001$ & $<0.001$ & -0.009 & -0.230 & 0.818 & $<0.001$ & $<0.001$ \\
\hline
\end{tabular}

SE, standard error冈Cl, confidence interval.

Table 4 Association between categorical variables and times of hospitalization by multiple linear regression 


\begin{tabular}{|c|c|c|c|c|c|c|c|}
\hline \multirow[t]{2}{*}{ Variables } & \multirow[t]{2}{*}{ B } & \multirow[t]{2}{*}{ SE } & \multirow[t]{2}{*}{$B C$} & \multirow[t]{2}{*}{$t$} & \multirow{2}{*}{$\begin{array}{l}p \text { for } \\
\text { trend }\end{array}$} & \multirow{2}{*}{$\begin{array}{l}\text { Lower } \\
95 \% \mathrm{Cl}\end{array}$} & \multirow{2}{*}{$\begin{array}{l}\text { Upper } \\
95 \% \mathrm{Cl}\end{array}$} \\
\hline & & & & & & & \\
\hline \multicolumn{8}{|l|}{ Type } \\
\hline 1 & reference & & & & & & \\
\hline 2 & -0.084 & 0.472 & -0.007 & -0.178 & 0.859 & -1.011 & 0.843 \\
\hline \multicolumn{8}{|l|}{ Gender } \\
\hline Female & reference & & & & & & \\
\hline Male & -0.017 & 0.111 & -0.006 & -0.151 & 0.880 & -0.235 & 0.202 \\
\hline \multicolumn{8}{|l|}{ Smoking } \\
\hline Yes & reference & & & & & & \\
\hline No & 0.282 & 0.141 & 0.093 & 2.001 & 0.046 & 0.005 & 0.560 \\
\hline Cessation & 0.455 & 0.206 & 0.103 & 2.213 & 0.027 & 0.051 & 0.859 \\
\hline \multicolumn{8}{|l|}{ Treatment } \\
\hline Insulin & reference & & & & & & \\
\hline NIM & -0.152 & 0.158 & -0.042 & -0.960 & 0.338 & -0.462 & 0.159 \\
\hline Insulin+ NIM & -0.148 & 0.125 & -0.053 & -0.189 & 0.235 & -0.394 & 0.097 \\
\hline GS & 0.209 & 1.410 & 0.006 & 0.148 & 0.882 & -2.559 & 2.977 \\
\hline \multicolumn{8}{|l|}{ Reasons } \\
\hline Diabetic foot infection & reference & & & & & & \\
\hline Poor glycemic control & -0.081 & 0.335 & -0.015 & -0.241 & 0.810 & -0.739 & 0.578 \\
\hline DKA & -0.074 & 0.855 & -0.004 & -0.087 & 0.931 & -1.753 & 1.604 \\
\hline HHS & -0.741 & 1.029 & -0.029 & -0.720 & 0.472 & -2.762 & 1.280 \\
\hline Hypoglycemia & -0.125 & 0.474 & -0.013 & -0.264 & 0.792 & -1.056 & 0.806 \\
\hline Infection & 0.193 & 0.308 & 0.048 & 0.628 & 0.530 & -0.411 & 0.798 \\
\hline Microalbuminuria & 0.259 & 1.430 & 0.007 & 0.181 & 0.856 & -2.549 & 3.068 \\
\hline Edema & 0.728 & 0.367 & 0.113 & 1.984 & 0.048 & 0.007 & 1.449 \\
\hline Blurred vision & -0.349 & 0.398 & -0.046 & -0.877 & 0.381 & -1.132 & 0.433 \\
\hline Dizziness & 0.044 & 0.300 & 0.012 & 0.146 & 0.884 & -0.546 & 0.633 \\
\hline Headache & -0.598 & 0.596 & -0.044 & -1.004 & 0.316 & -1.768 & 0.572 \\
\hline
\end{tabular}




\begin{tabular}{|c|c|c|c|c|c|c|c|}
\hline Chest distress & -0.741 & 0.634 & -0.051 & -1.169 & 0.243 & -1.986 & 0.504 \\
\hline Precordial discomfort & 0.593 & 0.634 & 0.041 & 0.935 & 0.350 & -0.652 & 1.837 \\
\hline Palpitation & 0.393 & 0.452 & 0.042 & 0.868 & 0.386 & -0.496 & 1.281 \\
\hline Dyspnea & -0.352 & 0.427 & -0.041 & -0.823 & 0.411 & -1.191 & 0.487 \\
\hline Chest pain & -0.741 & 1.430 & -0.021 & -0.518 & 0.605 & -3.549 & 2.068 \\
\hline Extremities pain & -0.341 & 0.390 & -0.047 & -0.874 & 0.382 & -1.106 & 0.425 \\
\hline Arthralgia & 0.593 & 0.855 & 0.029 & 0.693 & 0.488 & -1.086 & 2.271 \\
\hline Numbness & -0.306 & 0.398 & -0.041 & -0.768 & 0.443 & -1.089 & 0.477 \\
\hline Paresthesia & -0.074 & 0.855 & -0.004 & -0.087 & 0.931 & -1.753 & 1.604 \\
\hline Low back pain & -0.574 & 0.634 & -0.039 & -0.906 & 0.365 & -1.819 & 0.671 \\
\hline $\begin{array}{l}\text { Consciousness } \\
\text { disorders }\end{array}$ & -0.530 & 0.421 & -0.064 & -1.261 & 0.208 & -1.356 & 0.296 \\
\hline Abdominal distention & -0.574 & 0.634 & -0.039 & -0.906 & 0.365 & -1.819 & 0.671 \\
\hline Table 4 Cont. & & & & & & & \\
\hline \multirow[t]{2}{*}{ Variables } & \multirow[t]{2}{*}{ B } & \multirow[t]{2}{*}{ SE } & \multirow[t]{2}{*}{$\mathrm{BC}$} & \multirow[t]{2}{*}{$t$} & $p$ for & Lower & Upper \\
\hline & & & & & trend & $95 \% \mathrm{Cl}$ & $95 \% \mathrm{Cl}$ \\
\hline Abdominal pain & -0.202 & 0.474 & -0.020 & -0.427 & 0.670 & -1.133 & 0.729 \\
\hline Vomiting & -0.191 & 0.414 & -0.024 & -0.460 & 0.645 & -1.004 & 0.623 \\
\hline Diarrhea & -0.491 & 0.565 & -0.039 & -0.868 & 0.386 & -1.601 & 0.619 \\
\hline Constipation & 0.259 & 1.430 & 0.007 & 0.181 & 0.856 & -2.549 & 3.068 \\
\hline Urinary incontinence & 2.259 & 1.430 & 0.064 & 1.580 & 0.115 & -0.549 & 5.068 \\
\hline Melena & -0.741 & 1.029 & -0.029 & -0.720 & 0.472 & -2.762 & 1.280 \\
\hline Pruritus & 0.188 & 0.463 & 0.020 & 0.406 & 0.685 & -0.720 & 1.096 \\
\hline Weakness & -0.225 & 0.322 & -0.048 & -0.699 & 0.485 & -0.858 & 0.408 \\
\hline Hepatic dysfunction & 0.259 & 0.855 & 0.013 & 0.303 & 0.762 & -1.419 & 1.938 \\
\hline Elevated blood glucose & -0.741 & 1.430 & -0.021 & -0.518 & 0.605 & -3.549 & 2.068 \\
\hline $\begin{array}{l}\text { The classic symptoms } \\
\text { of diabetes mellitus }\end{array}$ & -0.116 & 0.443 & -0.013 & -0.261 & 0.794 & -0.986 & 0.754 \\
\hline
\end{tabular}

GS+ insulin, GS+ NIM, no treatment and eye pain, mental disorders, limb movement disorders, dysuria, exanthema, shock, cough, gingival hemorrhage, toothache, appetite loss were missing correlations with 
insulin and diabetic foot infection, respectively.

\section{Figures}

\section{All patients with principal discharge diagnosis of diabetes $=5767$}

Excluded:

at admission in other departments $=426$

admitted in 2014 and discharged in $2015=29$

Admission and discharge were in endocrinology department. $(n=5312)$

Twice or more hospitalizations $(n=1721)$

Figure 1

Screening of readmitted diabetic patients 


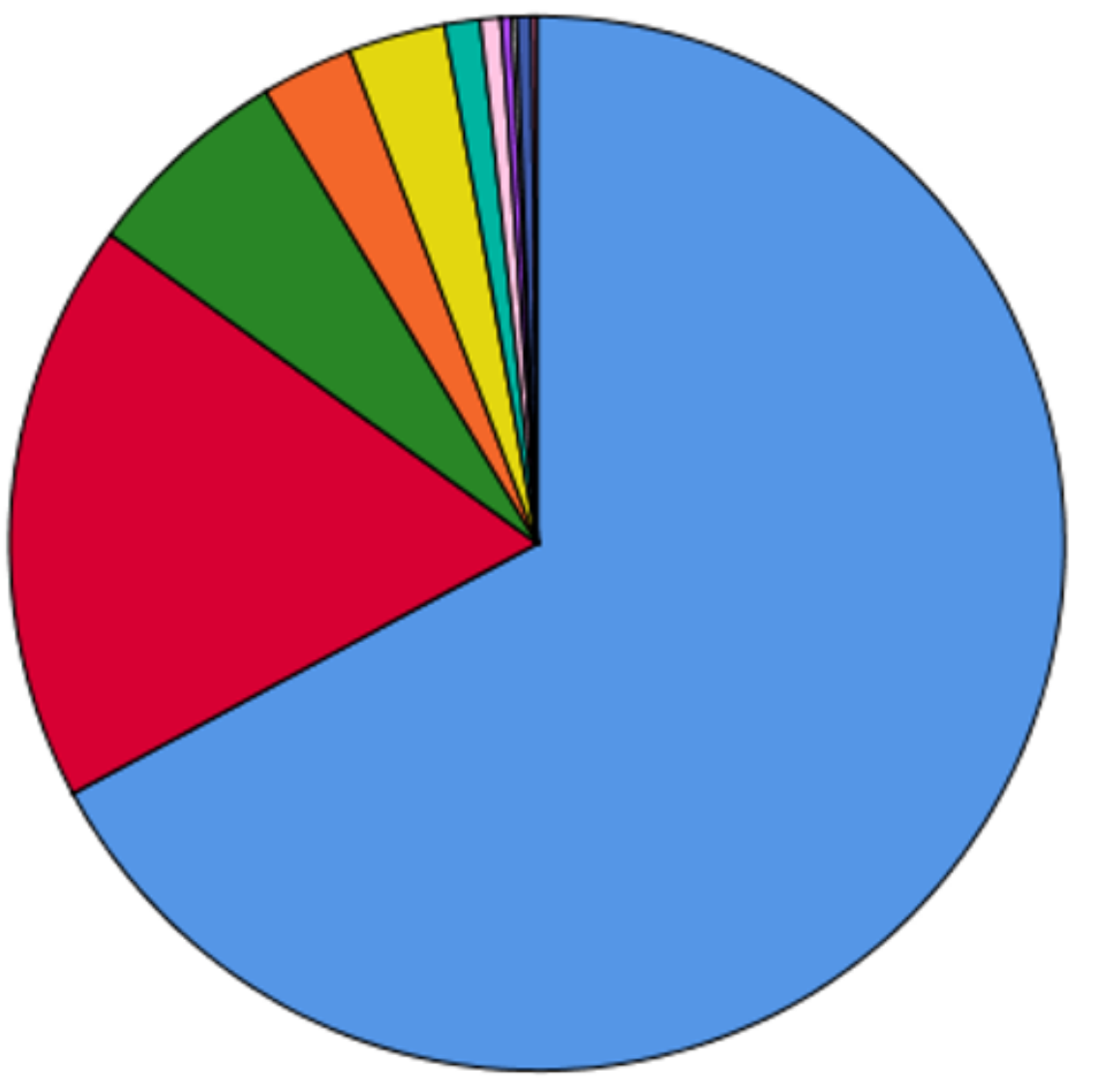

times

$\square 2$

$\square 3$

$\square 4$

$\square 5$

$\square 6$

$\square 7$

$\square 8$

$\square 9$

$\square 10$

$\square 11$

$\square 12$

Figure 2

Distribution of readmission times of diabetic patients in five years 